\title{
INDUSTRIAL EXPERIENCE WITH OXYGEN \\ CONTROL OF A FED-BATCH FILAMENTOUS FUNGAL FERMENTATION
}

\author{
L. Bodizs*, N. Faria ${ }^{+}$, M. Titica ${ }^{\dagger}$, B. Srinivasan* \\ H. Jorgensen ${ }^{+}$, D. Bonvin* and D. Dochain\# \\ *École Polytechnique Fédérale de Lausanne, \\ Lausanne, Switzerland \\ ${ }^{+}$Novozymes A/S, Bagsvaerd, Denmark \\ \# CESAME, Université Catholique de Louvain, \\ Louvain-la-Neuve, Belgium \\ ${ }^{\dagger}$ Laboratoire de Génie des Procédés, Centre de Recherche et \\ de Transfert de Technologie, Saint-Nazaire Cedex, France
}

\begin{abstract}
Fed-batch filamentous fungal fermentations at the industrial level are operated today either in an open-loop manner or through simple PI control, both with limited performance. The main challenge presented by such an operation is that, due to the presence of uncertainties of the inoculum, the process can go into oxygen limitation that severely affects production. In this paper, a cascade control strategy for regulation of the dissolved oxygen is presented, which incorporates available auxiliary measurements to improve performance. This strategy is formulated based on the investigation of the structural elements of a simplified process model developed from experimental data. Experimental results confirm the efficiency of the proposed control strategy. Copyright (c)2005 IFAC
\end{abstract}

Keywords: Biological processes, filamentous fungal fermentation, closed-loop operation, cascade control, model predictive control.

\section{INTRODUCTION}

Filamentous fungi are among the most frequently used cell factories in the fermentation industry. Their success is due to the relatively well established fermentation technology and to the versatility of strains available, allowing the production of a wide variety of products: primary metabolites, antibiotics, enzymes and proteins (McIntyre et al., 2001).

Traditionally, filamentous fungal fermentations in industry are operated in fed-batch mode (Titica et al., 2004). The feed rates are manipulated open-loop with or without manual intervention. Also, the biomass concentration is considerably higher than in other biological process. As a result of the filamentous structure of the biomass, high biomass concentration induces high viscosity, making oxygen transfer difficult (Li et al., 2000). In addition to difficulties with oxygen transfer, there are other factors such as the initial properties of the biomass (inoculum) that brings to a situation where there is insufficient oxygen for the biomass to continue its normal operation. Then, oxygen limitation occurs resulting in a decreased performance of the microorganisms. The goal of this work is to come up with a strategy that reduces the risk of running into oxygen limitation.

Manual control and simple PI controllers have been used for dissolved oxygen control on this process, but the range in which the dissolved oxygen could be confined is fairly wide. One possibility would be to operate with a sufficiently high set- 
point for the oxygen concentration so that, despite these large variations, oxygen limitation does not occur. However, this corresponds to feeding less substrate and thus low production, which is unacceptable. Hence, the need for tight control of oxygen concentration at a reasonably low setpoint so that i) oxygen limitation can be avoided and ii) production is not sacrificed. It is important to note the operational and optimization objective behind this project, though only the issue of tight oxygen control will be addressed in this paper.

The poor performance of a PI controller (or manual control) can be attributed to the fact that it only uses the information on the variable to be controlled, though much more information is available via auxiliary measurements such as the oxygen uptake rate $(O U R)$ and carbon dioxide evolution rate $(C E R)$. The idea here is to incorporate some of these measurements in the control algorithm. For this purpose, the traditional and systematic way is to use a model-based controller with soft-sensors employed to estimate the states and the control input computed via optimization using the entire state information (Eaton and Rawlings, 1990). So, a simplified tendency model is developed with the parameters identified from experimental data. A model-based controller with software sensors is then designed. Also, by investigating the structure of the model, a simple cascade control scheme is proposed, where the outer-loop oxygen controller provides the setpoint for the $O U R / C E R$ signal that is controlled in the inner loop.

Both algorithms are tested experimentally. It is seen that the level of modeling is insufficient to provide improved performance with the modelbased controller. On the other hand, the simple cascade controller proposed is quite effective in keeping the dissolved oxygen in a very tight range.

The paper is organized as follows. In the next section, the process under consideration and its operation in industry are described. In Section 3, the first-principle model of the process is briefly reviewed. In Section 4, the feedback control scheme is detailed. The outcome of the experiments, carried out at pilot-scale level at Novozymes, Bagsvaerd, Denmark, are presented in Section 5. Conclusions in Section 6 close the paper.

\section{PROCESS DESCRIPTION AND INDUSTRIAL PRACTICE}

\subsection{Fungal Fermentation}

The process studied in this paper is $\alpha$-amylase production by Aspergillus Oryzae. The same substrate (glucose $s$ ) is consumed for both growing the biomass $(x)$ and for producing the enzyme $(p)$.
The main problem this process presents is oxygen limitation where there is insufficient dissolved oxygen $(D O)$ in the liquid phase. This is usually caused by high biomass concentration that, due to its filamentous structure, increases viscosity and makes oxygen transfer difficult.

\subsection{Current Operation}

The typical way of operating the process at Novozymes is presented in Figure 1. For the sake of confidentiality, the experimental results are normalized and so no measurement units are presented. The feeding policy takes into account the problem of oxygen limitation and it consists of:

(1) a batch phase or growth phase, during which the substrate concentration is reduced from a high initial value to its operational domain,

(2) a linearly-increasing feed rate whose role is to avoid oxygen limitation in the early phase of the fed-batch, and

(3) a constant feed rate that is chosen in order to exactly fill the reactor in the remaining operation time and keep the dissolved oxygen without bounds.

It can be observed that the initial substrate concentration is high, in order to produce a lot of biomass during the batch phase (growth phase), which is expected to increase process performance due to its catalyzing role.

As can be seen from Figure 1, the range of dissolved oxygen is fairly wide in the operating interval (20-40\%). Also, towards the end of the batch, increasing the feed rate causes oxygen limitation. Higher feed rate means more biomass, higher viscosity and reduced oxygen transfer.

Also, it is important to note that the $D O$ level drops drastically due to a runaway phenomenon. This phenomenon has been encountered and analyzed by the biologists in Novozymes, and the inhouse knowledge can be explained as follows. Once under oxygen limitation, the biomass tries to react to this situation to avoid suffocation. Thus, the filaments spread in an attempt of finding a zone with higher DO level (they 'reach out for air'). This spread, however, increases significantly the viscosity of the medium and decreases the oxygen transfer even more. This positive feedback causes the runaway phenomenon, which in turn leads to lower and lower oxygen levels and probably death of microorganisms.

So, it is clear that, if a tight control of oxygen is not put in place, the risk of running into oxygen limitation is very high. Therefore, it is necessary to study the possibilities of combatting uncertainties and avoiding oxygen limitation in order to maintain reproducible production levels. 

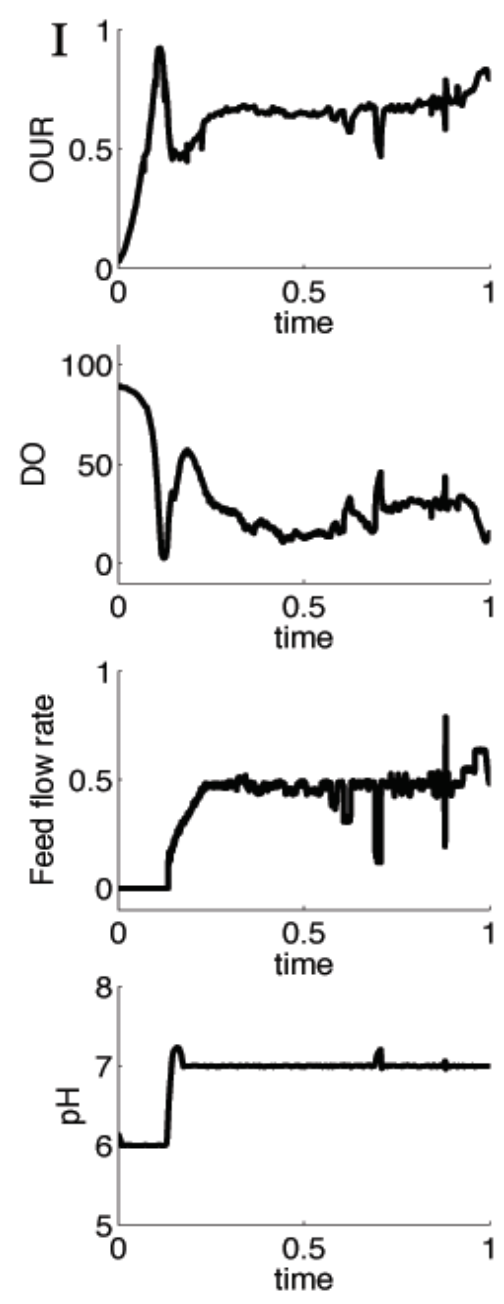

Fig. 1. Experiment I - current operation, $s(0)=$ 0.9 .

\section{MODELING}

The biomass, due to its filamentous structure, is divided into three regions (Agger et al., 1998):

- active region $\left(x_{a}\right)$ - responsible for production

- extension region $\left(x_{e}\right)$ - responsible for growth

- hyphal region $\left(x_{h}\right)$ - corresponding to the inactive part of the biomass.

The macroscopic reactions read:

$$
\begin{aligned}
& s+D O \stackrel{x_{a}}{\rightarrow} x_{e} \\
& s+D O \stackrel{x_{e}}{\rightarrow} x_{a} \rightarrow x_{h} \\
& s+D O \stackrel{x_{e}}{\rightarrow} p
\end{aligned}
$$

A first-principle model of the process was built with the aim of developing a control scheme (Titica et al., 2004). Here, only the dynamic mass balance equations are given, necessary to justify the control structure presented in Section 4. The algebraic equations $\left(\mathcal{F}_{i}, i=\{1,2, \ldots, 6\}\right)$ can be found in (Titica et al., 2004).

Morphological states

$$
\begin{aligned}
& \dot{x}_{e}=q_{1}-\frac{F}{V} x_{e}, \quad x_{e}(0)=x_{e 0} \\
& \dot{x}_{a}=q_{3}-q_{1}-q_{2}-\frac{F}{V} x_{a}, \quad x_{a}(0)=x_{a 0} \\
& \dot{x}_{h}=q_{2}-\frac{F}{V} x_{h}, \quad x_{h}(0)=x_{h 0}
\end{aligned}
$$

where $q_{1}=\mathcal{F}_{1}$ is the rate of extension (branching), $q_{2}=\mathcal{F}_{2}$ the rate of inactivation, $q_{3}=\mathcal{F}_{3}$ the growth rate, $F$ the feed rate, and $V$ the volume.

Glucose

$$
\begin{aligned}
\dot{s}=-\left(Y_{x s} q_{3}\right. & \left.+Y_{p s} r_{p s} x_{a}+m_{s}\left(x_{a}+x_{e}+x_{h}\right)\right) \\
& +\frac{F}{V}\left(s_{f}-s\right), \quad s(0)=s_{0}
\end{aligned}
$$

where $r_{p s}=\mathcal{F}_{4}$ is the specific rate of enzyme production, $m_{s}$ the maintenence coefficient, and $s_{f}$ the feed concentration. $Y_{x s}$ and $Y_{p s}$ are the yield coefficients for substrate consumption for growth and production, respectively.

Enzyme

$$
\dot{p}=r_{p s}-\frac{F}{V} p, \quad p(0)=p_{0}
$$

Dissolved oxygen

$$
\begin{gathered}
\dot{D O}=-r_{D O}\left(x_{a}+x_{e}+x_{h}\right)+k_{L} a\left(D O^{*}-D O\right) \\
-\frac{F}{V} D O, \quad D O(0)=D O_{0}
\end{gathered}
$$

where $r_{D O}=\mathcal{F}_{5}$ is the specific rate of oxygen consumption, $k_{L}=\mathcal{F}_{6}$ the gas-liquid mass transfer coefficient, $a$ the transfer area, and $D O^{*}$ the equilibrium $D O$ level.

Volume

$$
\dot{V}=F-F_{\text {evap }}, \quad V(0)=V_{0}
$$

where $F_{\text {evap }}$ stands for the water evaporation rate.

All model parameters, except the ones related to the growth of the individual biomass components, were fitted to industrial data provided by Novozymes. The growth parameters could not be fitted since biomass components were not measurable individually. The measurements used for model fitting were i) on-line: $D O$, viscosity (included in $\mathcal{F}_{6}$ ), OUR, CER, $V$ and ii) off-line: enzyme level $p$ and quantity of total biomass $\left(x=x_{a}+x_{e}+x_{h}\right)$.

\section{CONTROLLER DESIGN}

As mentioned above, simple output controllers for $D O$ control do not provide sufficient level of performance. So, additional measurements are sought 
to be used to improve performance. The auxiliary measurements that are available for this process are $p H$, pressure, viscosity, $V, O U R$ and $C E R$. Since $p H$ and pressure have not been incorporated in the model and the viscosity sensor is not always reliable, the idea is to come up with a control strategy that uses $O U R, C E R$ and $V$ measurements.

\subsection{Model-based Predictive Controller}

The traditional way of incorporating additional measurements is through a software sensor followed by control input computation (either analytically or via optimization) (Eaton and Rawlings, 1990).

In the software sensor part, the process model developed in the previous section is used to predict the states of the model in some open-loop manner (Dochain, 2003). As some of the states $(D O$ and $V)$ and variables $(O U R$ and $C E R$ ) are measurable, the corresponding equations are simply omitted and the states and variables replaced with the measurements.

For the computation of the control input (feed flow rate), an optimization-based approach is used (Bemporad, 1998). To be cautious, the predicted dissolved oxygen trajectory is also extracted from the optimization routine, and a simple controller is used to reduce the difference between this and the measured trajectories.

\subsection{Cascade Controller}

Interesting features can be seen by analyzing the structure of oxygen dynamics. The feed rate has at least two main effects on the dissolved oxygen: (i) an immediate effect through dilution (the last term on the right hand side of (4)) - increasing $F$ decreases $D O$, and (ii) integral effect through oxygen consumption (first term) - increasing $F$ increases the substrate in the reactor and thus the consumption, which in turn decreases DO. The influence of $F$ on the transfer between the gaseous and the liquid phases (second term) is quite negligible compared to the other two terms.

If the DO level is controlled only through dilution, as simple linearizing or output controllers do, the accumulation of substrate causes a further dip in $D O$, which gives rise to oscillations and poor performance. This is typical of systems where the zero dynamics plays an important role in the process performance (Mullhaupt et al., 1999).

This problem can be better understood by considering the linearized version of a problem with both direct and integral effects: $\dot{x}_{1}=-a x_{2}-u$ and $\dot{x}_{2}=u$, where $a$ is a factor that represents the relative importance of the direct and integral terms. In transfer function form, $\frac{X_{1}(s)}{U(s)}=-\frac{s+a}{s^{2}}$. The characteristic polynomial with a proportional controller $-k$ is $s^{2}+k s+a k$ which will have complex roots if $k<4 a$. So, in the presence of a fast zero ( $a$ large), one needs to use a very large gain to get non-oscillatory performance. Such a large gain may not be admissible due to other couplings and nonlinearities of the problem.

Though including derivative action is an option to improve performance, it is not really feasible due to the large amount of noise in the measurements. But, fortunately $r_{D O} x$ can be 'measured' via $O U R$ and $C E R$ measurements. So, the idea is to use $r_{D O} x$ as a manipulated variable to control the dissolved oxygen and $F$ to control $r_{D O} x$. This leads to the cascade structure of Figure 2, in which the role of the inner loop is to regulate the substrate consumption based on $O U R$ and $C E R$ measurements, while the outer loop gives the setpoint for this consumption.

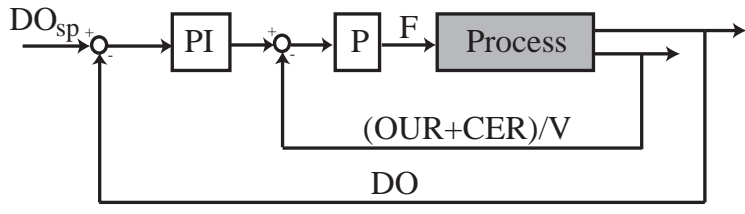

Fig. 2. Cascade controller for dissolved oxygen

Going back to the simple linear example, $\dot{x}_{1}=$ $-a x_{2}-u$ and $\dot{x}_{2}=u$, the proposed cascade control (without integral action) is equivalent to $u=k_{i}\left(-k_{o}\left(x_{1, \text { ref }}-x_{1}\right)-x_{2}\right)$, which corresponds to a full state feedback $\left(k_{i}\right.$ is the inner-loop gain and $-k_{o}$ the outer-loop gain). The characteristic polynomial is then $s^{2}+k_{i}\left(k_{o}+1\right) s+a k_{i} k_{o}$, whose poles can be placed anywhere. Thus, the proposed cascade control is one way of implementing a state feedback for the part of the dynamics that concerns dissolved oxygen.

\section{EXPERIMENTAL VERIFICATION OF THE PROPOSED SCHEMES}

The results from two experiments are presented in Figures 3 and 4.

\subsection{Performance of Model-based Controller}

The model-based controller is tested during the first phase of Experiment II. Though the model was fit to give good one-step ahead predictions, and fairly reasonable multi-step simulations, the optimization drove the system to unrealistic scenarios. The reasons for such a behavior could be numerous, ranging from accuracy of the model to 
local minima of the optimization routine. More insight needs to be obtained to critically evaluate the shortcomings of this controller.
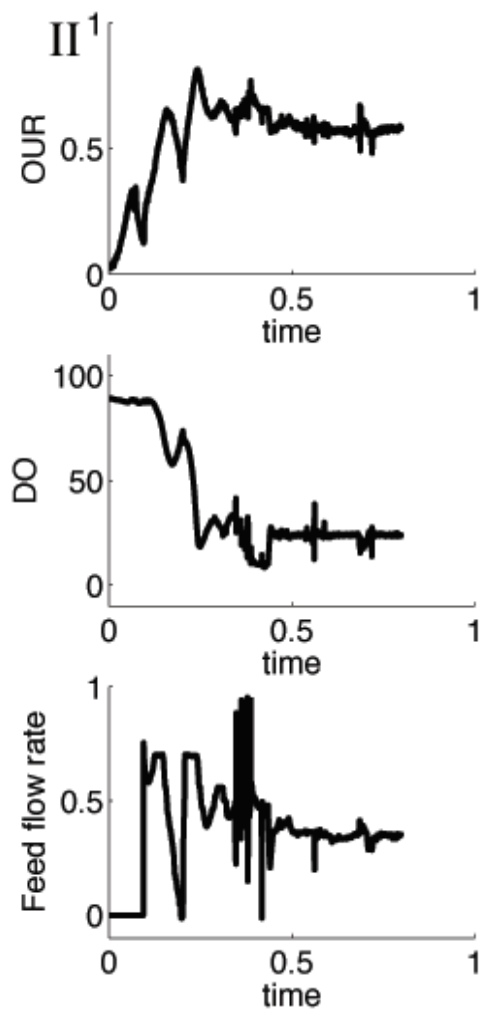

Fig. 3. Experiment II - model-based predictive control followed by cascade control, $s(0)=$ 0.089 .

\subsection{Performance of Cascade Controller}

Since the model-based controller did not give reasonable performance, it was decided to switch on the cascade controller with constant setpoint after 0.45 time units in Experiment II. The glitch around 0.6 time units is caused by a sensor failure and the dip near 0.7 time units is due to a deliberate change in setpoint. It can be seen that the setpoint tracking is quite satisfactory. Again, in Experiment III, the cascade controller is put in place after 0.07 time units and worked satisfactorily until 0.75 time units, as explained in Section 5.4. The deviation from the setpoint in the operational interval is $2 \%$, which is a major improvement compared to the usual operation which had a range of $20 \%$.

\subsection{Initial Substrate Concentration}

As can be seen in Figure 1, the dissolved oxygen goes to zero, even before the feeding starts. This is due to the high initial substrate concentration which invariably pushes the system to oxygen
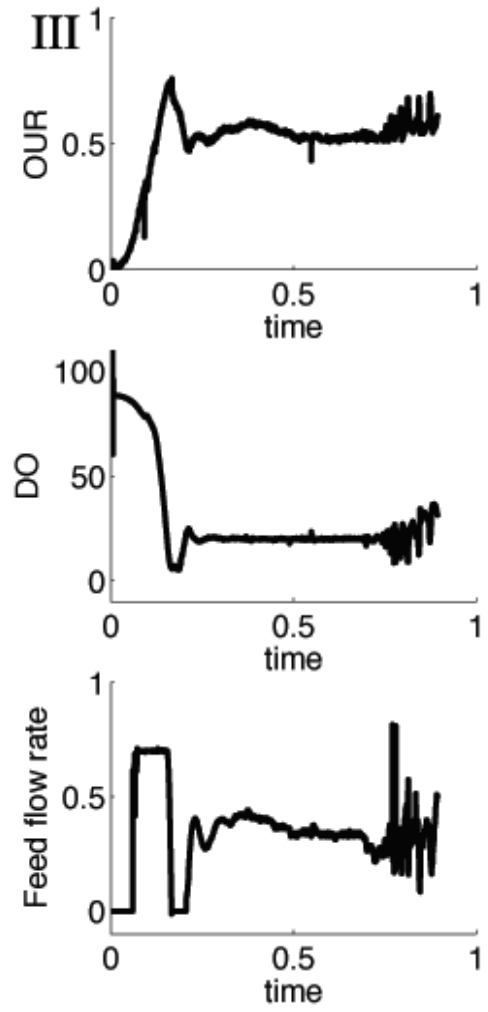

Fig. 4. Experiment III - cascade control, $s(0)=$ 0.089 .

limitation at the end of the batch phase. So, the idea is to reduce $s_{0}$ to avoid this problem.

Also, simulation studies on the developed model predicted that the same amount of enzyme could be produced with a lower value of $s_{0}$. This is because, by reducing $s_{0}$, only the amount of inactive biomass is reduced, while the amount of active biomass remains unchanged (Bodizs et al., 2004). In addition, the model suggested that a lower $s_{0}$ is good for oxygen control since growing less biomass in turn decreases the viscosity of the medium, thus leading to better oxygen transfer. So, the experiments were carried out with $s_{0}$ being one-tenth of its usual value.

However, the experimental results indicate that, with reduced initial substrate concentration, the production is only two-thirds of its usual value. The reason might be due to the effect of $p H$, that has not been considered in the model. Note that in practice (as can be seen in Figure 1), the medium is slightly acidic during growth phase, while it is neutral during production. As a consequence, growth requires condition that are different from those required for production. As the amount of substrate available during the growth phase is considerably reduced by lowering $s_{0}$, very little biomass is formed. Thus, the production phase start with a small amount of biomass, that is insufficient to produce the required amount of enzyme. 


\subsection{Setpoint for Dissolved Oxygen}

Once a good controller is put in place, an important decision is to choose an appropriate setpoint for the dissolved oxygen. Once again, we turned to the model to provide the setpoint, which gives a setpoint smaller than that in Experiment II. The setpoint provided by the model is considered to be below the norm and risky in industrial standards, and the experimental results confirmed the same.

This setpoint is implemented in Experiment III and the controller worked satisfactorily for most of the time. But the ringing behavior, typical of oxygen limitation, started to occur after 0.75 time units. Once this mode is entered, the phenomena of oxygen limitation being highly nonlinear, the linear cascade control failed to pull the system out of it.

\subsection{Transients}

Though the regulatory performance of the controller is very good, the transients show an overshoot. This is due to saturation of the input and other factors not being properly accounted for in the control design. Other strategies, such as controlling the $O U R$, can be applied since it is a more reliable indication of biomass activity than $D O$ at an early phase of the batch. These are some issues that need to be looked into in the near future.

\section{CONCLUSION}

The main outcome of this study is an efficient control scheme for the dissolved oxygen. The proposed cascade controller effectively regulates the $D O$ level and a proper setpoint needs to be chosen to keep the system away from oxygen limitation. The advantage of the proposed control strategy is that it is simple to apply and not specific to the strain of microorganism studied in this paper. Thus, it is applicable to a wide variety of fungal fermentations.

This study indicated that the model developed has several shortcomings. It predicts a feasible operation with a low initial substrate concentration and a low dissolved oxygen setpoint, both of which could not be experimentally verified. Also, a model-based optimization approach does not yield satisfactory results.

In the next experiments, the initial substrate concentration could be increased to some intermediate values. A strategy for $\mathrm{pH}$ control needs to be formulated to guarantee a certain level of biomass growth, and thereby certain production levels. Another open challenge is to chose the best setpoint or reference profile for the dissolved oxygen.

Acknowledgements: This paper presents the results of the Knowledge-driven Batch Production (BatchPro) European Project HPRN-CT-200000039 .

\section{REFERENCES}

Agger, T., B. Spohr, M. Carlsen and J. Nielsen (1998). Growth and roduct formation of aspergillus oryzae during submerged cultivations: verification of a morphologically structured model using fluorescent probes. Biotech. Bioeng. 57, 321-329.

Bemporad, A. (1998). Reducing conservatism in predictive control of constrained systems with disturbances. In: 37th IEEE CDC. Tampa, FL, USA. pp. 1384-1389.

Bodizs, L., B. Srinivasan and D. Bonvin (2004). Optimal feeding strategy for bioreactors with biomass death. In: IFAC CAB9. Nancy, France.

Dochain, D. (2003). State and parameter estimation in chemical and biochemical processes : a tutorial. J. Process Contr. 13, 801-818.

Eaton, J. W. and J. B. Rawlings (1990). Feedback control of nonlinear processes using on-line optimization techniques. Comp. Chem. Eng. 14, 469-479.

Li, Z. J., V. Shukla, A. P. Fordyce, A. G. Pedersen, K. S. Wenger and M. R. Marten (2000). Fungal morphology and fragmentation behavior in fed-batch aspergillus oryzae fermentation at the production scale. Biotech. Bioeng. 70, 300-312.

McIntyre, M., C. Muller, J. Dynesen and J. Nielsen (2001). Metabolic engineering of the morphology of aspergillus. Adv. Biochem. Eng./Biotech. 73, 104-128.

Mullhaupt, P., B. Srinivasan, J. Levine and D. Bonvin (1999). Cascade control of the toycopter. In: ECC'99. Karlsruhe, Germany.

Titica, M., L. Bodizs, F. Lei, B. Srinivasan, D. Dochain and D. Bonvin (2004). Modeling and optimization of fed-batch filamentous fungal fermentation. In: BatchPro Symposium. Poros, Greece. pp. 55-65. 